\title{
Refractive-index measurements with a Pellin-Broca prism apparatus
}

\author{
K. Schmid and A. Penzkofer
}

\begin{abstract}
An experimental system is described for measuring refractive indices of liquids and solids over a wide spectral range from the near IR to the near UV. A Pellin-Broca prism is used as the dispersing element. The obtained accuracy is $\Delta n= \pm 10^{-4}$.
\end{abstract}

\section{Introduction}

The absolute phase refractive index $n$ relates the phase velocity $v$ in matter to the vacuum light velocity $c(n=c / v)$. It is determined by the molecular polarizability of the medium and gives insight to the interaction of light with matter and the structure of substances. ${ }^{1-3}$ Additionally the group refractive index $n_{g}$ is needed to describe light pulse propagation, ${ }^{4,5}$ which is defined as the ratio of vacuum light velocity to group velocity $v_{g}$ in matter ${ }^{6}\left(n_{g}=c / v_{g}\right)$.

Various techniques have been developed to measure the refractive index $n$ (for reviews see Refs. 2, 7, and 8). For liquids and solids most methods use the refraction or total reflection of light beams at prisms. The refractive indices of gases are generally measured with interferometers. The spectral range of commercially available refractive-index instruments is often limited to the sodium $D$ line (wavelength $\lambda_{D}=589.3 \mathrm{~nm}$ ) or some discrete lines of spectral lamps in the visible. The group refractive index $n_{g}$ may be calculated from the wavelength dependence of the refractive index $n(\lambda)$. Direct measurements of the group refractive index are scarce $^{9}$

In this paper we describe a versatile apparatus for determining the phase refractive indices of liquids and solids. The wavelength may be continuously varied. Measuring phase refractive indices at closely spaced frequencies allows accurate calculation of the group refractive index. Various light sources such as tungsten halogen lamps, high-pressure xenon or mercury arc lamps, spectral lamps, or lasers may be used. A gen-

The authors are with Universitat Regensburg, Naturwissenschaftliche Fakultat II-Physik, D-8400 Regensburg, Federal Republic of Germany.

Received 2 February 1983.

0003-6935/83/121824-04\$01.00/0.

1983 Optical Society of America. eralized Pellin-Broca prism is used as the dispersing element (hollow prism for liquids; prisms have to be formed from substances under investigation for solids). A spectrometer is used to select single frequencies for the measurements. The refracted light is detected with a silicon diode array detector. The 350-1100-nm spectral range is covered with a tungsten halogen lamp and visible optics. The spectral range may be extended down to $220 \mathrm{~nm}$ with xenon or mercury arc lamps and UV optics.

\section{Method}

The light passage through a generalized Pellin-Broca prism of arbitrary refracting angles $\alpha$ and $\beta$ is depicted in Fig. 1. The light rays are refracted at the entrance and exit faces and totally reflected on the intermediate surface. The angles $\alpha$ and $\beta$ are chosen nearly equal. Their magnitude is selected according to the range of refractive indices that should be covered (see below). In a restricted sense one speaks of Pellin-Broca prisms ${ }^{4}$ when $\alpha=\beta=30^{\circ}$.

In the following we restrict our discussion to the propagation of linearly polarized light through liquids, isotropic solids, and uniaxial crystals with the $c$ axis perpendicular to the electric field strength (ordinary rays). Within the cited limits the angular deviation $\delta$ of light is calculated by application of Snell's law of refraction at the entrance and exit faces and the law of reflection at the opposite side. The result is

$$
\begin{aligned}
\delta= & 90^{\circ}-\beta+\alpha-\varphi_{I} \\
& +\arcsin \left(n_{r}\left\{\sin \left[\alpha+\beta-\arcsin \left(\frac{1}{n_{r}} \sin \varphi_{I}\right)\right]\right\}\right),
\end{aligned}
$$

where $n_{r}=n / n_{A}$ is the relative refractive index, $n$ is the absolute phase refractive index of the substance under investigation, and $n_{A}$ is the absolute refractive index of the surrounding air. Equation (1) allows $n_{r}$ to be calculated when angles $\alpha$ and $\beta$ of the Pellin-Broca prism, the ray deviation angle $\delta$, and the entrance angle $\varphi_{I}$ are known. A computer program is used to determine $n_{r}$ 


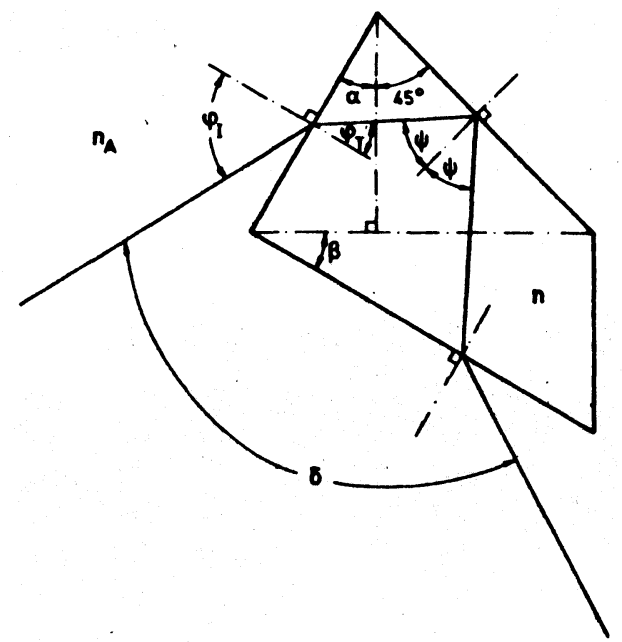

Fig. 1. Ray tracing through a general Pellin-Broca prism: $n_{A}$, absolute phase refractive index of air; $n$, absolute phase refractive index of liquid or solid.

from the measured incident angle $\varphi_{I}(\delta, \alpha$, and $\beta$ are fixed).

If $\delta=90^{\circ}$ and $\alpha=\beta$, Eq. (1) reduces to

$$
\varphi_{I}=\arcsin \left(n_{r}\left\{\sin \left[2 \alpha-\arcsin \left(\frac{1}{n_{r}} \sin \varphi_{I}\right)\right]\right\}\right),
$$

which has a solution for

$$
\sin \varphi_{I}=n_{r} \sin \alpha .
$$

Equation (3) is Snell's law for the symmetric path of light rays through the Pellin-Broca prism $\left(\alpha=\varphi_{T}\right)$.

The dependence of the relative refractive index $n_{r}=$ $n / n_{A}$ on the entrance angle $\varphi_{I}$ is plotted in Fig. 2 for various prism angles $\alpha=\beta$ and an angular deviation $\delta$ $=90^{\circ}$ (Eq. (2) or (3)]. Large $\alpha$ angles give high resolution $\left(n_{r}\right.$ varies slowly with $\left.\varphi_{I}\right)$, but the refractiveindex range is limited (small upper limits of $n_{r}$ ). The selection of $\alpha$ depends on the expected range of refractive indices to be measured.

The accuracy of the refractive-index measurement may be estimated by calculating the variation of $n_{r}$ with changes of $\varphi_{I}$. From Eq. (3) one finds

$$
\Delta n_{r}=\frac{\cos \varphi_{1}}{\sin \alpha} \Delta \varphi_{I}
$$

$\Delta n_{r}$ is small (high accuracy) for large entrance angles $\varphi_{I}$ and large refracting angles $\alpha$.

The absolute phase refractive index $n=n_{r} n_{A}$ and the group refractive index $n_{g}$ at a fixed vacuum wavelength $\lambda$ or frequency $\nu(\nu=c / \lambda)$ are connected by the relation $^{4}$

$$
n_{g}=\frac{n}{1+\frac{\lambda}{n} \frac{\partial n}{\partial \lambda}}=\frac{n}{1-\frac{\nu}{n} \frac{\partial n}{\partial \nu}} .
$$

Measurement of $n$ at two closely spaced wavelengths $\lambda_{1}$ and $\lambda_{2}$ allows one to calculate the group refractive index at $\lambda=\left(\lambda_{1}+\lambda_{2}\right) / 2$. (Here $\partial n / \partial \lambda$ is replaced by $\left[n\left(\lambda_{1}\right)\right.$ $\left.-n\left(\lambda_{2}\right)\right] /\left(\lambda_{1}-\lambda_{2}\right)$.

The absolute refractive index of air depends somewhat on atmospheric pressure and temperature. ${ }^{10}$ The

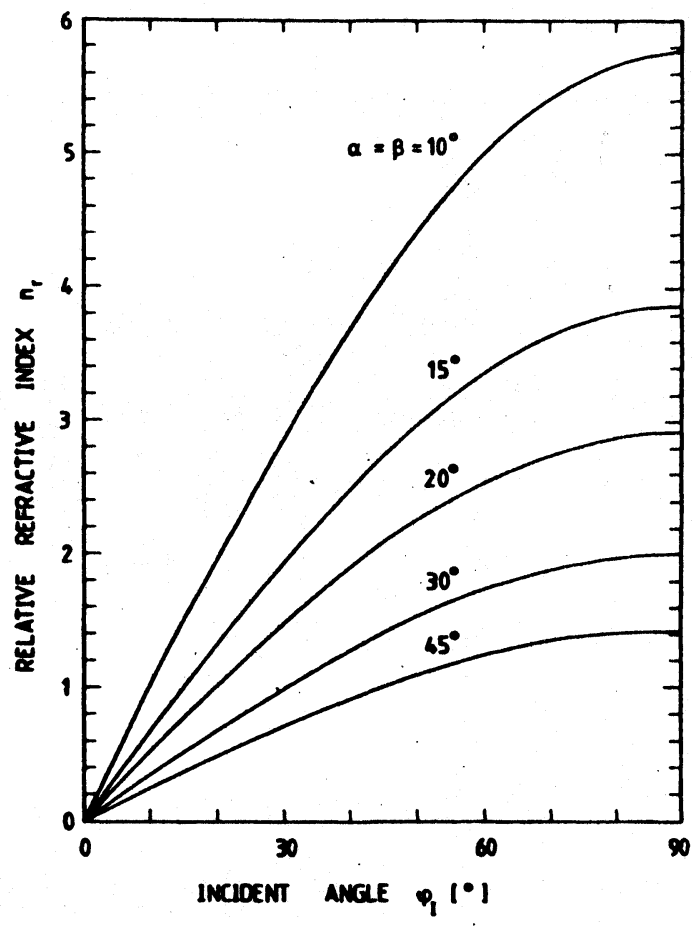

Fig. 2. Relative refractive index $n_{r}$ vs incident angle $\varphi_{I}$ of light to Pellin-Broca prism for various refracting angles $\alpha=\beta$. Deflection angle $\delta=90^{\circ}$.

following refractive-index dispersion equation of air (dry, with 0.03 vol. \% $\mathrm{CO}_{2}$ ) is rewritten from Ref. 10:

$$
\begin{aligned}
n_{A}(\tilde{\nu}, t, p)= & 1+\left(6.4328 \times 10^{-5}+\frac{2.94981 \times 10^{6}}{1.46 \times 10^{10}-\tilde{\nu}^{2}}+\frac{2.554 \times 10^{4}}{4.1 \times 10^{9}-\tilde{\nu}^{2}}\right) \\
& \times \frac{p_{A}\left(1.054915+8.3 \times 10^{-4} p_{A}-1.2423 \times 10^{-5} p_{A} t_{A}\right)}{1.013877+3.7118 \times 10^{-3} t_{A}}
\end{aligned}
$$

where $\tilde{\nu}=\nu / c$ is the frequency in $\mathrm{cm}^{-1}, t$ is the temperature in ${ }^{\circ} \mathrm{C}$, and $p$ is the pressure in bars $\left(n_{A}=\right.$ 1.0002678 at $\lambda_{D}=589.3 \mathrm{~nm}, t_{A}=25^{\circ} \mathrm{C}$, and $p_{A}=1 \mathrm{~atm}$ $=1.01325$ bar .

\section{Experimental}

The experimental setup for the refractive-index measurement is depicted in Fig. 3: (a) the system for conventional light sources (tungsten halogen lamps, spectral lamps, xenon, or mercury arc lamps); (b) the experimental arrangement for laser sources ( $\mathrm{cw}$ or pulsed).

The system with conventional light sources [Fig. 3(a)] uses an $\mathrm{He}-\mathrm{Ne}$ laser to facilitate alignment. The alignment procedure is as follows. The He-Ne laser is focused to the slit $S 1$ (0.1-mm width) with a cylindrical lens $C L$ (focal length $f=20 \mathrm{~cm}$ ), while lamp $L$ is removed. The light is collimated with lens $L 1(f=1.33$ m). the distance between $S_{1}$ and $L_{1}$ is equal to the focal length of $L 1$. A right-angle prism $P$ directs the light beam to the Pellin-Broca prism. To set a fixed deflection angle of $\delta=90^{\circ}$ the Pellin-Broca prism is replaced by a penta prism whose deviation from $\delta=90^{\circ}$ is $<3 \mathrm{sec}$ of arc. Lens $L 2(f=1 \mathrm{~m})$ focuses the light 

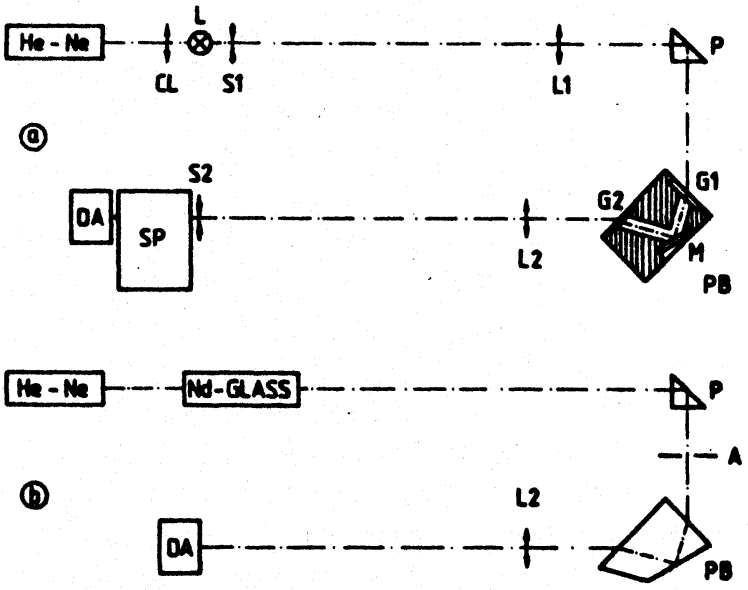

Fig. 3. Experimental setup for refrective-index measurement with (a) conventional light sources and (b) laser light sources. He-Ne, Ho-Ne laser; $C L$, cylindrical lens $(f=20 \mathrm{~cm}) ; L$, light source; $S 1$, slit (0.1-mm width); $L 1$, achromatic lens $(f=1.33 \mathrm{~m}) ; P, 90^{\circ}$ prism; $P B$, Pellin-Broca prism (1-cm hole diam); G1, G2, Suprasil windows; $M$, aluminum front mirror; L2, achromatic lens $(f=1 \mathrm{~m}) ; S 2$, slit (0.03-mm width); $S P$, spectrometer $(f=25 \mathrm{~cm})$ with grating of 1200 lines/mm; DA, silicon diode array (Tracor DARSS). Nd:glase, mode-locked Nd:glase laser; $A$, aperture.

pulse to the entrance slit $S 2(0.03-\mathrm{mm}$ width) of the spectrometer. It is mounted on a translation stage to set the L2-S2 distance equal to the focal length of $L 2$ (adjusted for each wavelength; at focal distance the detected signal is constant, independent of lateral passage through lens $L 2$ ). The $25-\mathrm{cm}$ spectrometer (1200-1/mm grating) is mounted on a translation stage for fine positioning perpendicular to the optical axis of $L 2$. After adjusting the $90^{\circ}$ deviation angle $\delta$, the penta prism is removed, and the Pellin-Broca prism is inserted. The support consists of a precision $360^{\circ}$ rotation table (resolution $0.001^{\circ}$, Micro-Control type RT 120) mounted on two crossed tilt platforms and a translation stage (movement perpendicular to optical axis of $L 2$ ). The Pellin-Broca prism is rotated so that the $\mathrm{He}-\mathrm{Ne}$ laser is reflected back to slit S1, and the zero position of the incident angle $\varphi_{I}$ is obtained. Lamp $L$ is now inserted. The Pellin-Broca prism is rotated until the wanted spectral component passes through slit S2 and is detected at the silicon diode array $D A$ (Tracor DARSS-system) behind the spectrometer. To compensate concentric errors of the rotation table the angle $\varphi_{I}$ is measured four times, whereby the start angle is changed by $\sim 90^{\circ}$ for each measurement. (Maximum deviation from average value was found to be $0.003^{\circ}$.) The refractive index v8 wavelength may be recorded continuously when tungsten lamps, xenon, or mercury high-pressure arc lamps are used. Measuring the light wavelength behind slit $S 2$ for two slightly different incident angles $\varphi_{I}$ (difference $0.010^{\circ}$ ), the group refractive indices are easily determined [Eq. (4)].

Accurate measurement of refractive indices requires a precise determination of the angles $\alpha$ and $\beta$ of the Pellin-Broca prism. For the investigation of liquids the hollow Pellin-Broca prism form was drilled into a superrefined steel cylinder as indicated in Fig. 3(a). Plane-parallel plates $G_{1}$ and $G_{2}$ of fused silica are used as windows, and a plane-parallel aluminum mirror $M$ is used as reflector. The parallelism of the plates and the mirror was measured with an autocollimation telescope. The small wedge of the plates ( $\leq 3 \mathrm{sec}$ of arc) was positioned perpendicular to the plane spanned by the light path so that the plates are exactly parallel with the plane of refraction and reflection. The angles $\alpha$ and $\beta$ are determined by measuring the angles between $G 1$, $M$ and $G 1, G 2$ with the rotation stage and the autocollimation telescope. Values of $\alpha=30.142^{\circ} \pm 0.001^{\circ}$ and $\beta=29.723^{\circ} \pm 0.001^{\circ}$ were obtained for our sample. For the refractive-index measurement of solids a PellinBroca prism has to be cut out of the same material and polished. The angles $\alpha$ and $\beta$ are measured in the same way as for the hollow prism. The accuracy of the $90^{\circ}$ deflection angle $\delta$ was tested with a plane mirror mounted on the rotation stage instead of the PellinBroca prism. An angle of $\delta=\left(90 \pm 5 \times 10^{-4}\right)^{\circ}$ was measured.

The alignment of the system of Fig. 3(b) for the measurement of refractive indices at laser frequencies with $\mathrm{Cw}$ or pulsed lasers is similar to the procedure described above. Again the position of $\delta=90^{\circ}$ beam deflection at the diode array is detected by insertion of a $90^{\circ}$ pentaprism. The experimental arrangement is simpler since the entrance laser beam is already parallel. The refracted and reflected beam is directly focused on the diode array because the laser light is already monochromatic. A 1-mW He-Ne laser was attenuated and used as a cw light source. The system was also tested with a 5-psec pulse from a mode-locked Nd:glass laser.

The refractive index of liquids changes strongly with temperature. ${ }^{2}$ For this reason the hollow Pellin-Broca prism is thermostated by temperature stabilized water circulation through a cylindrical enclosure.

Compared with the technique of minimum deviation through a triangular dispersion prism, the technique described here has the advantage of fixed $90^{\circ}$ deviation. Only the Pellin-Broca prism has to be rotated, all other elements are fixed. The Pellin-Broca prisms are as easily fabricated as triangular dispersion prisms.

\section{Results}

Continuous light sources allow spectral continuous measurement of refractive indices and calculation of group refractive indices. The absolute accuracy of the measured refractive index is determined by the accuracy of the measurement of the involved angles and the slit widths of $S_{1}$ and $S_{2}$. The angles $\alpha$ and $\beta$ were measured to an accuracy of $\pm 0.001^{\circ}$. The slit $S_{2}$ of the spectrometer accepts light within an angle of $\Delta \delta=0.0017^{\circ}$ (= slit width/focal length). The accuracy of the rotation table is $\pm 0.001^{\circ}$, which gives an error of $\Delta \varphi_{I}=$ $\pm 0.001^{\circ}$. Insertion of these error values into Eq. (1) gives an error of the refractive index of $\Delta n= \pm 9 \times 10^{-5}$ for $\varphi_{I}=45^{\circ}$ and $\alpha=\beta=30^{\circ}$. The error reduces for larger angles of $\varphi_{I}, \alpha$, and $\beta$. Changes of refractive in- 
dices (e.g., due to wavelength or temperature variation) are detected with an accuracy of $\Delta n= \pm 2 \times 10^{-5}$.

To test the apparatus refractive indices of the liquids (water and carbon tetrachloride) and the glass (SF2) were measured at various frequencies between 350 and $1100 \mathrm{~nm}$. Obtained values at $\lambda=589.3 \mathrm{~nm}$, air temperature $t_{A}=22.5^{\circ} \mathrm{C}$, and pressure $p_{A}=980 \mathrm{mbar}$ were (1) for $\mathrm{H}_{2} \mathrm{O} n_{r}=1.3328, n=1.3331, n_{g}=1.3510(t=$ $24.5 \pm 0.5^{\circ} \mathrm{C}$, no special treatment of distilled water, (2) for $\mathrm{CCl}_{4} n_{r}=1.4608, n=1.4611, n_{g}=1.4886\left(t=20^{\circ} \mathrm{C}\right)$, and (3) for Schott glass SF2 $n_{r}=1.64754, n=1.64797$, and $n_{g}=1.7071$ (angle $\alpha=30.0486^{\circ}, \beta=30.0069^{\circ}$ ). The reported value is $n_{r}=1.64752$ (Schott data sheet).

\section{Conclusions}

The apparatus described allows accurate and easy measurement of refractive indices over a wide frequency region from the UV to the IR. The spectral range may be further extended by suitable light sources and detectors. Any refractive-index value may be measured by proper selection of refraction angles $\alpha$ and $\beta$ of the dispersing Pellin-Broca prism.
The authors thank T. Ascherl for technical assistance. They acknowledge financial support of the Deutsche Forschungsgemeinschaft.

\section{References}

1. S. S. Batsanov, Refractometry and Chemical Structure (Consultants Bureau, New York, 1961).

2. N. Bauer and K. Fajans, in Physical Methods of Organic Chemistry, Vol. 1, A. Weissberger, Ed. (Interscience, New York, 1945), p. 653.

3. A. Penzkofer, H. Glas, and J. Schmailzl, Chem. Phys. 40, 47 (1982).

4. E. Hecht and A. Zajac, Optics (Addison-Wesley, Reading, Mass., 1974).

5. R. Topp and G. C. Orner, Opt. Commun. 13, 276 (1975).

6. L. Brillouin, Wave Propagation and Group Velocity (Academic, New York, 1960).

7. H. Kessler, in Handbuch der Physik XVIII. Geometrische Optik, optische Konstante, optische Instrumente, H. Geiger and K. Scheel, Eds. (Springer, Berlin, 1927), p. 623.

8. G. E. Fishter, in Applied Optics and Optical Engineering, Vol. 5, Part 1, R. Kingslake, Ed. (Academic, New York, 1967), p. 363.

9. D. B. McDonald and S. A. Rice, Opt. Commun. 32, 416 (1980).

10. D. H. Rank, in Advances in Spectroscopy, Vol. 1, H. W. Thompson, Ed. (Interscience, New York, 1959), p. 76.

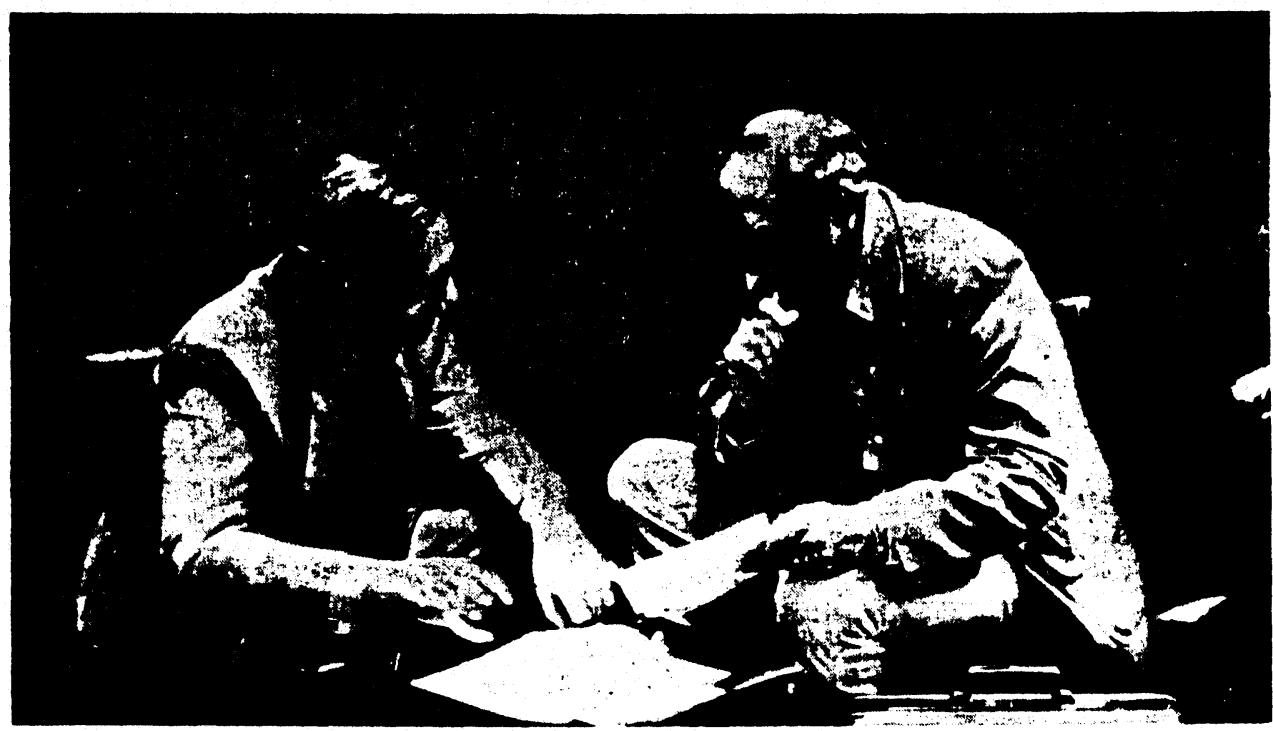

H. J. Bolle (Universitat Innsbruck) on the left and Thomas von der Haar (Colorado State University) photographed by P. Kopke (Meteorologishes Institut der Universitat Munchen) during the 1981 JAMAP meeting in Hamburg. 